\title{
Debulking mastectomy with electrochemotherapy: a case report of no surgery approach to recurrent breast cancer
}

\author{
Emanuela Esposito $^{1} \wedge$, Claudio Siani ${ }^{1}$, Ugo Pace $^{2}$, Raffaele Costanzo $^{3}$, Raimondo di Giacomo $^{1}$ \\ ${ }^{1}$ Breast Cancer Department, Istituto Nazionale Tumori di Napoli, IRCCS, Fondazione G. Pascale, Naples, Italy; ${ }^{2}$ Colorectal Cancer Surgery Unit, \\ Istituto Nazionale Tumori di Napoli, IRCCS, Fondazione G. Pascale, Naples, Italy; ${ }^{3}$ Thoracic Medical Oncology Unit, Istituto Nazionale Tumori di \\ Napoli, IRCCS, Fondazione G. Pascale, Naples, Italy \\ Correspondence to: Emanuela Esposito, MD, PhD, FEBS. Breast Cancer Department, Istituto Nazionale Tumori, IRCCS, Fondazione G. Pascale, via \\ Mariano Semmola, 52, 80131, Naples, Italy. Email: emanuela.esposito@istitutotumori.na.it; emanuelaexpo@hotmail.it.
}

\begin{abstract}
Electrochemotherapy has been shown to be safe, effective and non-invasive loco-regional treatment for chest wall breast cancer recurrence. Electrochemotherapy is a palliative treatment offered to patients with cutaneous metastases from breast cancer, which are not eligible for resection and/or systemic therapy is ineffective or contraindicated. Electrochemotherapy combines the administration of bleomycin with electroporation of tumor cell, intraoperatively. Here we present the case of a women affected by multidrug resistant metastatic synchronous solid cancers who refused radical mastectomy after being diagnosed with recurrent ulcerated right breast cancer. We first describe an extended indication to electrochemotherapy to treat breast cancer recurrence after previous breast conserving surgery. Electroporation-induced necrosis through electrochemotherapy replaced surgery and was delivered in 30 minutes at 5,000 $\mathrm{Hz}$ frequencies at $730 \mathrm{~V}$ by hexagonal needle under general anesthesia. The necrosis of the remaining breast resulted in a voluminous eschar that was easily removed few months after leaving the chest wall free from macroscopic disease turning in a "bladeless mastectomy". This kind of breakthrough application of electrochemotherapy might be considered to avoid palliative mastectomy in very selected patients. New technologies may help clinicians to find agreement between patient' will and the burden of treatment and might contribute in selected cases to give options to patients not keen on having surgery.
\end{abstract}

Keywords: Electrochemotherapy (ECT); breast cancer recurrence; case report; mastectomy; multidrug resistance

Submitted Aug 28, 2020. Accepted for publication Nov 27, 2020.

doi: $10.21037 /$ tcr-20-2803

View this article at: http://dx.doi.org/10.21037/tcr-20-2803

\section{Introduction}

The incidence of multiple primary malignant neoplasms has increased during the last decades as screening program has been implemented and cancer therapies have become more effective.

Since first described by Billroth in 1889 (1) and after reviewed by Warren and Gates in 1932 (2), specific guidelines have been drawn, however multiple neoplasms can translate in multiple drug resistance which drives clinicians to opt for different strategies (3).

Breast cancer is the most common cause of cancer amongst women (4). The risk of developing breast cancer increases with age. The gold standard for the treatment of early breast cancer is breast conserving surgery along with radiation therapy. The 15 -year absolute risk of breast cancer death is $3.8 \%$ (95\% CI: $1.6-6.0 \%, \mathrm{P}=0.00005)$. The 10 -year risk of any (loco-regional or distant) first recurrence is $19.3 \%$ if radiation treatment follows surgery (5).

In $5 \%$ to $30 \%$ local recurrence appears with synchro-

^ ORCID: 0000-0002-6394-8419. 
nous skin metastases (6). Skin metastases do not impact on overall survival, however, they have a major impact on the quality of life of patients causing pain, ulceration, infection, and psychological discomfort. Systemic treatments often fail to provide adequate local control of the disease. The most of patient are not eligible for resection and frequently develop resistance to any other systemic therapy (6).

Electrochemotherapy (ECT) has shown encouraging results amongst patients with skin metastases from breast and other types of solid cancers (7-9). ECT combines the administration of antineoplastic drugs such as cisplatin or bleomycin (6) with electroporation of tumor cells (6), intraoperatively. Electric pulses permeabilize the cell membrane and allow the drug to penetrate the cell and increase cytotoxicity. The codification of the procedure by European Standard Operating Procedure (ESOPE) guidelines in 2006 (10) led to a broad diffusion of the procedure, mainly in European centers, and since then, the progressive clinical experience, together with the emerging technologies, have extended its application to new groups of patients (7).

ECT is an effective palliative treatment of patients with cutaneous metastases $(6,7)$ who, due to the extent of their metastases, are not eligible for resection or radiotherapy has already been used, and systemic therapy is ineffective or contraindicated. Several clinical studies have demonstrated that ECT provides safe, effective, and non-invasive locoregional treatment for chest wall breast cancer recurrence. Few side effects have been reported such as skin irritation or rash, caused by the adhesives in the electrodes or the tape holding the electrodes in place $(11,12)$.

We present the following case in accordance with the CARE reporting checklist (available at http://dx.doi. org/10.21037/tcr-20-2803).

Here we present an extended indication to ECT for breast cancer recurrence after breast conserving surgery. Electroporation-induced necrosis replaced surgery and was delivered to treat breast cancer recurrence in multi-drug resistant patient who refused standard surgery. The effect was a massive necrosis of the remaining breast that resulted in a non-surgical mastectomy. This is one of a kind different application of ECT and gives the chance to avoid palliative surgery in very selected patients.

\section{Case presentation}

A 72-year-old woman with a personal history of breast, lung and rectal cancer presented with an ulcerated breast local recurrence on her right breast. She had no familial history of breast and ovarian cancer and refused any genetic testing. In 1994, when she was 46 and premenopausal, she was diagnosed with hormone receptor positive breast cancer, no special type (NST). She had breast conserving surgery and axillary clearance along with 25 applications of conventional external beam radiation therapy. Anatomic stage by TNM was pT2N0M0. She was prescribed 5 years endocrine therapy with tamoxifen and ovarian suppression with luteinizing hormone-releasing hormone (LHRH) agonists.

In 2010 she was diagnosed with right non-small cell lung cancer (NSCLC) complicated by pleural effusion. Stage at diagnosis was T2N1M1a. She was treated with firstline chemotherapy 4 cycles of cisplatinum and pemetrexed and obtained partial response. Ten months later, she had a local relapse. She was screened for epidermal growth factor receptor (EGFR) mutation and deletion of exon 19 (Ex19Del) was found. Thus, she started with EGFR tyrosine kinase inhibitor (TKI) gefitinib.

Since August 2013, following mediastinal lymph node progression, the patient was administered platinum-based doublet chemotherapy. After further progression, patient was treated with third line with EGFR TKI erlotinib—and targeted radiotherapy on lung lesion.

In 2015 she complained with abdominal pain. Rectal examination showed a rigid mass close to the dentate line. She underwent colonoscopy that revealed an ulcerated mass occupying half of the circumference of the colorectal lumen and rectal biopsy was performed. She was diagnosed with locally advanced rectal cancer and scheduled for neoadjuvant chemo-radiotherapy. She had complete response and refused completion rectal surgery.

In 2016 she developed right in-breast lump complicated by mediastinal lymphadenopathy. Mammography and ultrasound scan were suspicious for breast local relapse, then confirmed by right breast core biopsy showing receptor positive human epidermal growth factor receptor 2 (HER2) amplified breast cancer [estrogen receptor (ER) 85\%; progesterone receptor (PgR) 40\%; Ki67 20\%; HER2 +++]. She started weekly paclitaxel, anti-HER2 monoclonal antibody trastuzumab every 21 days and oral vinorelbine. She developed peripheral neuropathy, asthenia and dyspeptic syndrome, thus suspended paclitaxel and kept on with trastuzumab. Disease was deemed stable after 6-month follow-up.

In 2018, lung cancer progressed therefore patient underwent 45 Gy Cyber-knife System radiotherapy. The positron emission tomography-computed tomography (PET-CT) scan showed good response on the lung. 


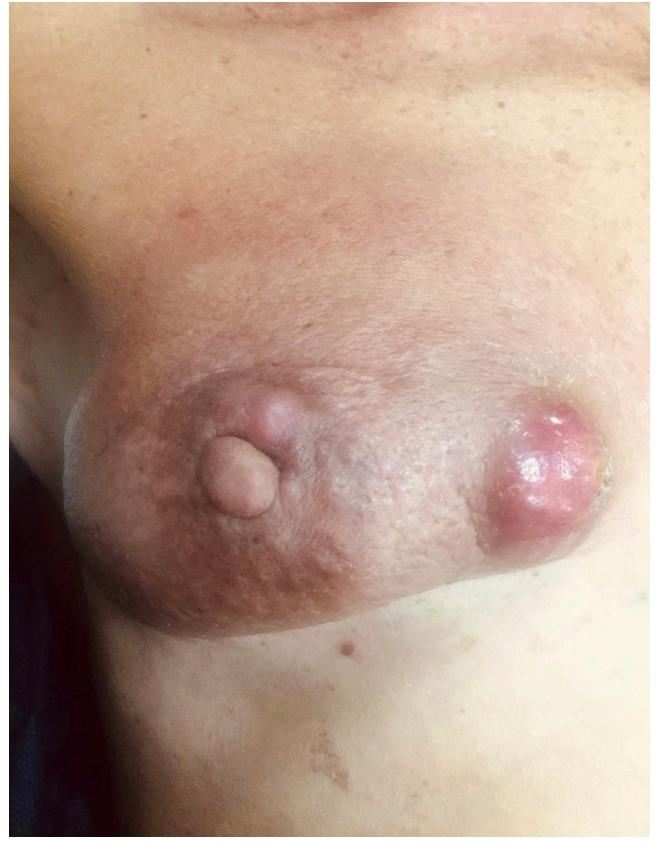

Figure 1 Preoperative image. Right breast cancer recurrence with skin ulceration, swelling and redness.

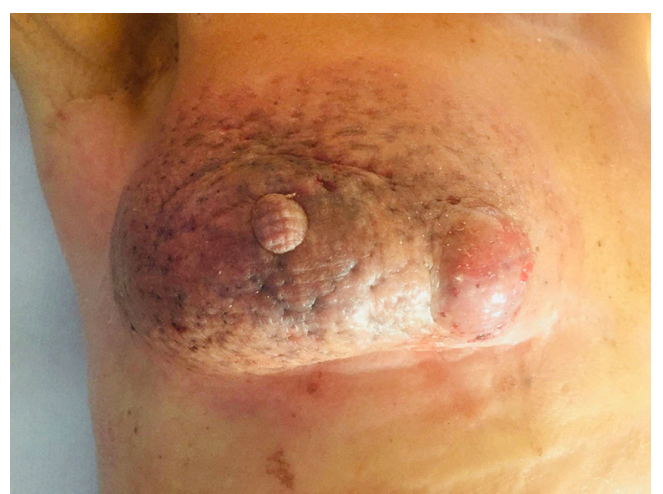

Figure 2 Post-operative image. Right breast 30 days after electrochemotherapy.

In 2019 rectal cancer recurred and underwent robotic Miles' abdominoperineal resection surgery and left colostomy. At the same time breast recurrence progressed with skin ulceration, redness and infiltration of pectoralis muscle (Figure 1).

The infiltrated area was $8-\mathrm{cm}$ large and extended all over the right hemi-thorax.

Multidisciplinary meeting (MDM) went through different surgical options. Modified radical mastectomy without tissue flap reconstruction was excluded as there was

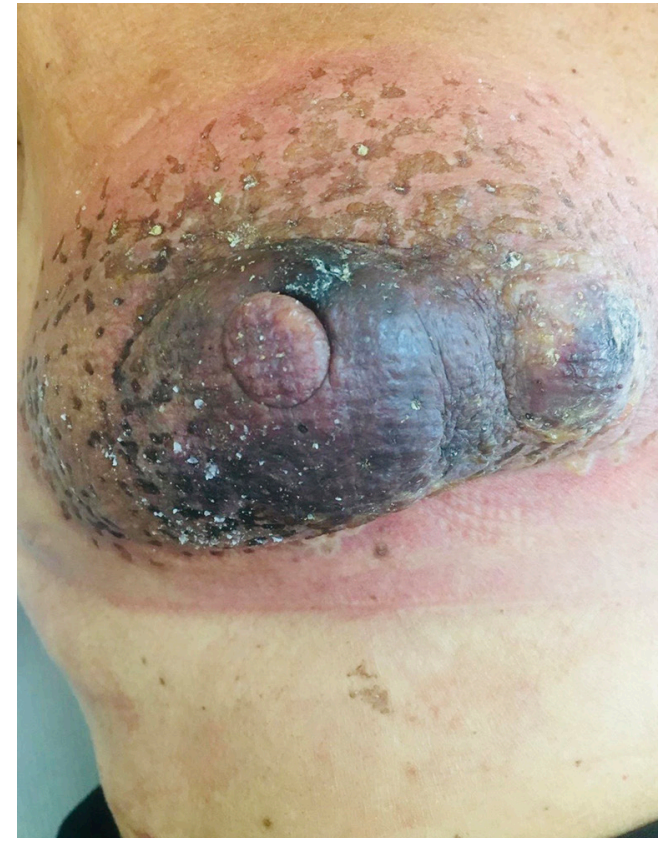

Figure 3 Post-operative image. Right breast 60 days after electrochemotherapy.

not enough skin to bridge the gap. Skin salvage mastectomy and reconstructive tissue flap was first offered to patient who refused that.

After a second MDM ECT on the breast was proposed. Patient was explained about risks and benefit of the procedure and signed the informed consent. She was scheduled for ECT on the right breast under general anesthesia. After intravenous injection of bleomycin $15 \mathrm{mg} / \mathrm{m}^{2}, 101$ pulses at $5,000 \mathrm{~Hz}$ frequencies were delivered by hexagonal needle electrode $40 \mathrm{~mm}$ length, at $730-\mathrm{V}$ voltage with Cliniporator ${ }^{\mathrm{TM}}$ [IGEA Srl, Carpi (MO), Italy]. The treatment was administered following the ESOPE guidelines (10). The procedure lasted 30 minutes. At the end of the procedure greasy meshed gauzes dressing were applied. After treatment patient was brought in the post-anesthetic recovery room and then to the ward. Patient was discharged the day after in good condition. The post-treatment follow-up was scheduled on the 7th, 15th, 30th, 60th and 90th day (Figures 2,3). After 90 days the whole breast was replaced by necrotic tissue as shown in Figure 4. Escharotomy to remove the necrotic tissue was performed under local anesthesia, leaving the chest wall free from macroscopic disease and the result was a "bladeless mastectomy" induced by ECT. In order to evaluate the residual burden of disease after escharotomy we performed 


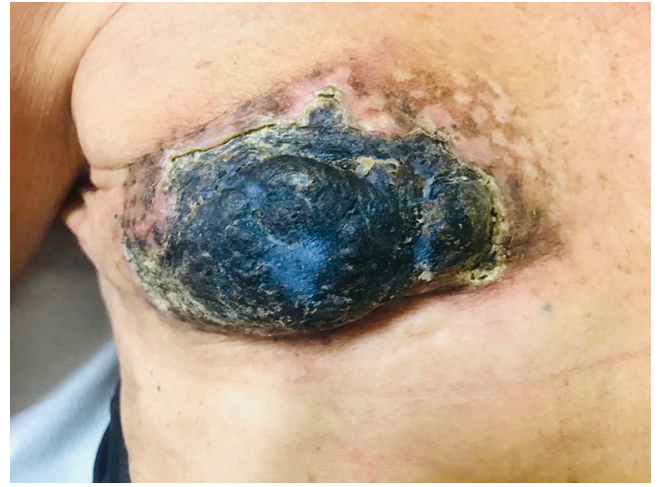

Figure 4 Post-operative image. Right breast 90 days after electrochemotherapy.

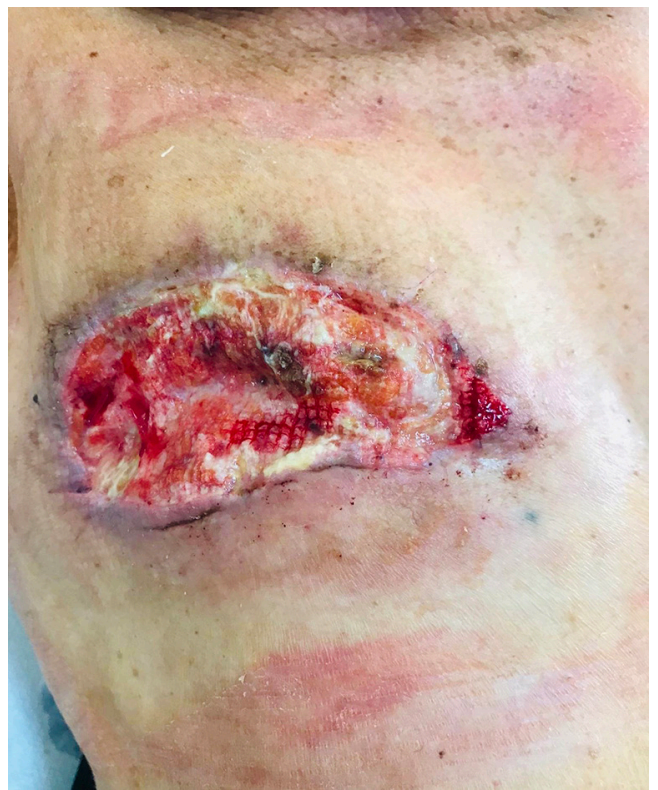

Figure 5 Post-operative image. Right side chest wall 1 day after escharotomy.

multiple punch biopsies on the living tissue underneath the eschar. Pathology report showed few residual of breast cancer cells surrounded by disease-free margins (Figure 5). Advanced dressings were applied during follow up. After 2 months the wound closed (Figure 6). Six months after the wound closed the patient was clinically free from disease on the chest wall (Figure 7). PET-CT scan showed non-evident disease from rectal cancer whereas lung metastases are stable. Patient is satisfied with the procedure and continues her routinely follow-up with improved quality of life.

All procedures performed in studies involving human

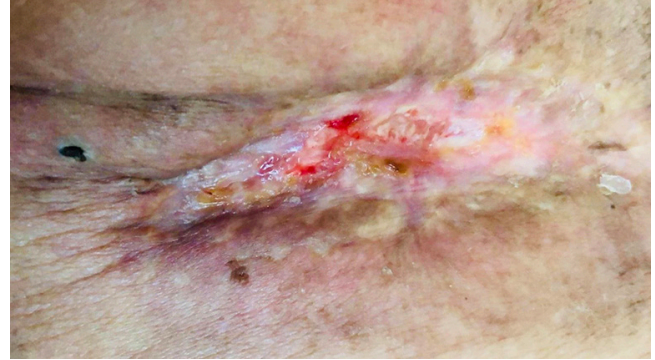

Figure 6 Wound closure sixty days after escharotomy.

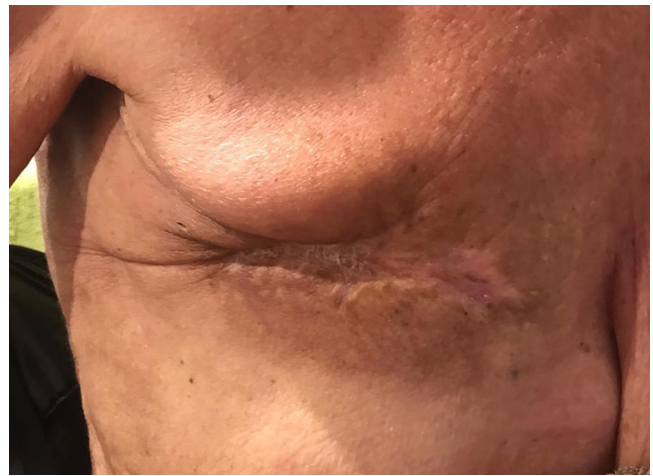

Figure 7 Wound closure 6 months after escharotomy.

participants were in accordance with the ethical standards of the institutional and/or national research committee(s) and with the Helsinki Declaration (as revised in 2013). Written informed consent was obtained from the patient for publication of this Case report and any accompanying images.

\section{Discussion}

It is not uncommon for people with severe cancer to refuse treatment, even when that decision might worsen their prognosis. However, new technologies may help clinicians to find agreement between patient' will and the burden of treatment. Here we have presented the case of a women treated for multiple primary cancers whose prognosis has been worsened by several local and distant relapses and resistant to different drugs. After more than 20 years of cancer fighting the patient had the right to refuse surgery consisting of mastectomy and flap reconstruction that she considered a major surgery to deal with. On the other hand, her quality of life was significantly impaired by ulcerative and exudative breast cancer recurrence that required daily dressing and frequently antibiotics cycling.

It is well-established ECT represent an effective option 
to treat patients with cutaneous metastases $(6,7)$ and it is now a breach of duty not to offer ECT to those patients. Nevertheless, this was not the case. Skin ulceration, malodorous and swelling lump need to be treated with mastectomy in order to improve patient quality of life. However, since the patient refused surgery we considered ECT as a possible option to replace debulking mastectomy. The electroporation-induced necrosis fulfilled our expectations by creating a voluminous eschar involving the whole breast, easily removed by escharotomy under local anesthesia. ECT is a fast procedure that can be easily delivered in about 30 minutes either under local and general anesthesia. The recovery is quick compared to major surgery such as breast and chest wall reconstruction. As far as we know, this is the first time ECT has replaced debulking mastectomy after breast conserving surgery. It has represented one of a kind breakthrough application of this technology in a very selected case from our group. Although someone can argue the healing process is quite long, the patient was greatly happy to have been spared from major surgery. She could change her dressing easily at home and felt very comfortable with the procedure. Thanks to this technology we gave a different option to very selected patient not keen on having breast surgery after recurrence.

\section{Acknowledgments}

Funding: None.

\section{Footnote}

Reporting Checklist: The authors have completed the CARE reporting checklist. Available at http://dx.doi.org/10.21037/ tcr-20-2803

Conflicts of Interest: All authors have completed the ICMJE uniform disclosure form (available at http://dx.doi. org/10.21037/tcr-20-2803). EE serves as an unpaid editorial board member of Translational Cancer Research from May 2020 to Apr 2022. The other authors have no conflicts of interest to declare.

Ethical Statement: The authors are accountable for all aspects of the work in ensuring that questions related to the accuracy or integrity of any part of the work are appropriately investigated and resolved. All procedures performed in studies involving human participants were in accordance with the ethical standards of the institutional and/or national research committee(s) and with the Helsinki Declaration (as revised in 2013). Written informed consent was obtained from the patient.

Open Access Statement: This is an Open Access article distributed in accordance with the Creative Commons Attribution-NonCommercial-NoDerivs 4.0 International License (CC BY-NC-ND 4.0), which permits the noncommercial replication and distribution of the article with the strict proviso that no changes or edits are made and the original work is properly cited (including links to both the formal publication through the relevant DOI and the license). See: https://creativecommons.org/licenses/by-nc-nd/4.0/.

\section{References}

1. Billroth T. Die allgemeine chirurgische pathologie and therapie. G. Reimer, 1889:14.

2. Warren S, Gates O. Multiple primary malignant tumors: a survey of the literature and a statistical study. Am J Cancer 1932;16:1358-414.

3. Markakis C, Marinis A, Dikeakos P, et al. Multiple synchronous primary neoplasms of the breast, colon and rectum after surgery for endometrial cancer: A case report. Int J Surg Case Rep 2013;4:493-5.

4. Cuzick J, Stewart H, Rutqvist L, et al. Cause-specific mortality in long-term survivors of breast cancer who participated in trials of radiotherapy. J Clin Oncol 1994;12:447-53.

5. Early Breast Cancer Trialists' Collaborative Group (EBCTCG), Darby S, McGale P, et al. Effect of radiotherapy after breast-conserving surgery on 10year recurrence and 15 -year breast cancer death: metaanalysis of individual patient data for 10,801 women in 17 randomised trials. Lancet 2011;378:1707-16.

6. Wichtowski M, Murawa D, Kulcenty K, et al. Electrochemotherapy in Breast Cancer - Discussion of the Method and Literature Review. Breast Care (Basel) 2017;12:409-14.

7. Campana LG, Edhemovic I, Soden D, et al. Electrochemotherapy - Emerging applications technical advances, new indications, combined approaches, and multi-institutional collaboration. Eur J Surg Oncol 2019;45:92-102.

8. Kunte C, Letulé V, Gehl J, et al. Electrochemotherapy in the treatment of metastatic malignant melanoma: a prospective cohort study by InspECT. Br J Dermatol 
2017;176:1475-85.

9. Bertino G, Sersa G, De Terlizzi F, et al. European Research on Electrochemotherapy in Head and Neck Cancer (EURECA) project: Results of the treatment of skin cancer. Eur J Cancer 2016;63:41-52.

10. Gehl J, Sersa G, Garbay J, et al. Results of the ESOPE (European Standard Operating Procedures on Electrochemotherapy) study: Efficient, highly tolerable and simple palliative treatment of cutaneous and

Cite this article as: Esposito E, Siani C, Pace U, Costanzo R, di Giacomo R. Debulking mastectomy with electrochemotherapy: a case report of no surgery approach to recurrent breast cancer. Transl Cancer Res 2021;10(2):1144-1149. doi: $10.21037 /$ tcr-20-2803 subcutaneous metastases from cancers of any histology. J Clin Oncol 2006;24:abstr 8047.

11. Matthiessen LW, Keshtgar M, Curatolo P, et al. Electrochemotherapy for Breast Cancer-Results From the INSPECT Database. Clin Breast Cancer 2018;18:e909-17.

12. Esmaeili N, Friebe M. Electrochemotherapy: A Review of Current Status, Alternative IGP Approaches, and Future Perspectives. J Healthc Eng 2019;2019:2784516. 